\title{
Ensemble of Local and Global Information for Finger-Knuckle-Print Recognition
}

\author{
Lin Zhang ${ }^{\mathrm{a}}$, Lei Zhang ${ }^{\mathrm{a}^{*}}$, David Zhang ${ }^{\mathrm{a}}$ and Hailong Zhu ${ }^{\mathrm{b}}$ \\ ${ }^{\mathrm{a}}$ Biometrics Research Center, Department of Computing, The Hong Kong Polytechnic University \\ ${ }^{\mathrm{b}}$ Research Institute of Innovative Product \& Technology, The Hong Kong Polytechnic University
}

Abstract. Biometrics authentication is an effective method for automatically recognizing a person's identity. Recently, it has been found that the finger-knuckle-print (FKP), which refers to the inherent skin patterns of the outer surface around the phalangeal joint of one's finger, has high capability to discriminate different individuals, making it an emerging biometric identifier. In this paper, based on the results of psychophysics and neurophysiology studies that both local and global information is crucial for the image perception, we present an effective FKP recognition scheme by extracting and assembling local and global features of FKP images. Specifically, the orientation information extracted by the Gabor filters is coded as the local feature. By increasing the scale of Gabor filters to infinite, actually we can get the Fourier transform of the image, and hence the Fourier transform coefficients of the image can be taken as the global features. Such kinds of local and global features are naturally linked via the framework of time-frequency analysis. The proposed scheme exploits both local and global information for the FKP verification, where global information is also utilized to refine the alignment of FKP images in matching. The final matching distance of two FKPs is a weighted average of local and global matching distances. The experimental results conducted on our FKP database demonstrate that the proposed local-global information combination scheme could significantly improve the recognition accuracy obtained by either local or global information and lead to promising performance of an FKP-based personal authentication system.

Keywords: Biometrics, finger-knuckle-print, local-global information combination

Corresponding author. Email: cslzhang@comp.polyu.edu.hk. This research is supported by the Hong Kong Polytechnic University ICRG (G-YH54) and the Ho Tung Fund (5-ZH52). 


\section{Introduction}

Recognizing the identity of a person with high confidence is a critical issue in various applications, such as e-banking, access control, passenger clearance, etc. The need for reliable user authentication techniques has significantly increased in the wake of heightened concerns about security, and rapid advancement in networking, communication and mobility [1]. Biometrics based methods, which use unique physical or behavioral characteristics of human beings, are of broad interest and have great potentials because of their high accuracy and convenience to use in the modern e-world. With the rapid development of computing techniques, in the past several decades researchers have exhaustively investigated the use of a number of biometric characteristics, including fingerprint, face, iris, palmprint, hand geometry, voice and ear, etc.

Among various kinds of biometric identifiers, hand-based biometrics has been attracting considerable attention over recent years. Fingerprint [2-6], palmprint [7-9], hand geometry [10, 11], hand vein [12], and inner-knuckle-print $[13,14]$ have been proposed and well investigated in the literature. Recently, it has been found that the image pattern of skin folds and creases in the outer finger knuckle surface is highly unique and thus can serve as a distinctive biometric identifier. Compared with fingerprint, the finger knuckle surface has some advantages as a biometric identifier. At first, it is not easy to be abraded since people usually hold stuffs with the inner side of the hand. In addition, unlike the use of fingerprint, there is no stigma of criminal investigation associated with the finger knuckle surface, so it can have a high user acceptance [15]. Thus, the finger knuckle feature has a great potential to be widely accepted as a biometric identifier. Some researchers have already done salient works in this field. Woodard and Flynn [16] are among the first scholars who exploited the use of finger knuckle surface in biometric systems. They set up a 3D finger back surface database with the Minolta 900/910 sensor. For feature extraction, they used the curvature based shape index to represent the finger back surface. Woodard's work makes a good effort to validate the uniqueness of outer finger surface as a biometric characteristic. However, the cost, size and weight of the Minolta 900/910 sensor limit the use of it in a practical biometric system, and the time-consuming 3D data acquisition and processing limit its use in real-time applications. Later, Kumar and Ravikanth [17] 
proposed a 2D finger-back surface based personal authentication system. With respect to the feature extraction, they resorted to some subspace analysis methods such as PCA, LDA and ICA. With their design, the acquisition device is doomed to have a large size because nearly the whole hand back area has to be captured, despite the fact that the finger knuckle area only occupies a small portion of the acquired image. Furthermore, subspace analysis methods may be effective for face recognition but they may not be able to effectively extract the distinctive line and junction features from the finger knuckle surface. In Kumar's later work [15], they adopted the robust line orientation code (RLOC) [9] to extract the local orientation information of the finger-back surface images.

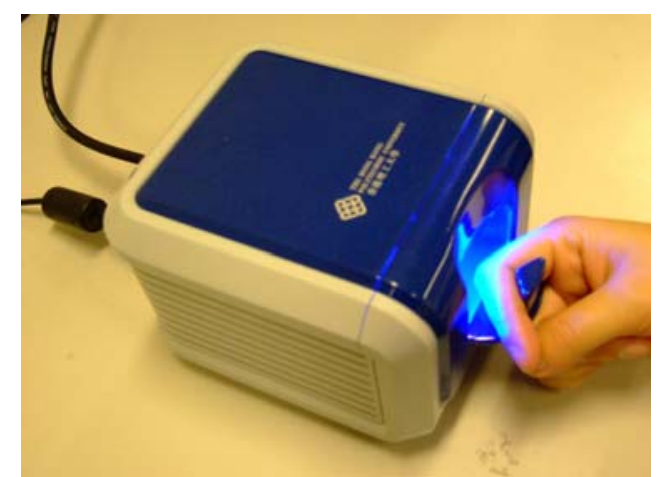

(a)

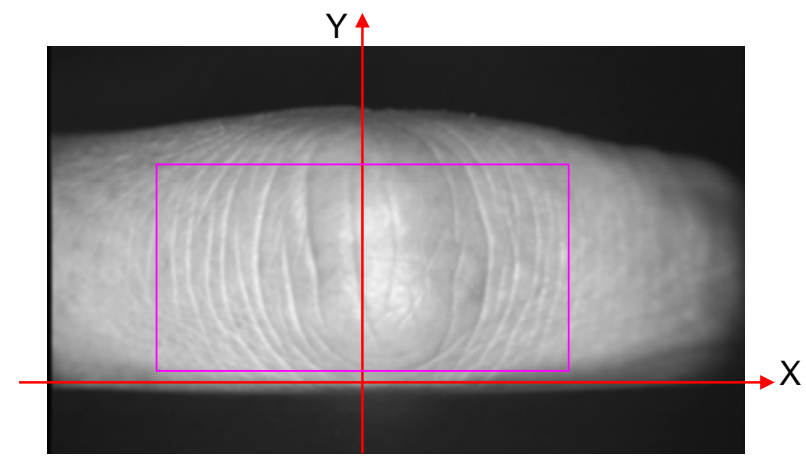

(c)

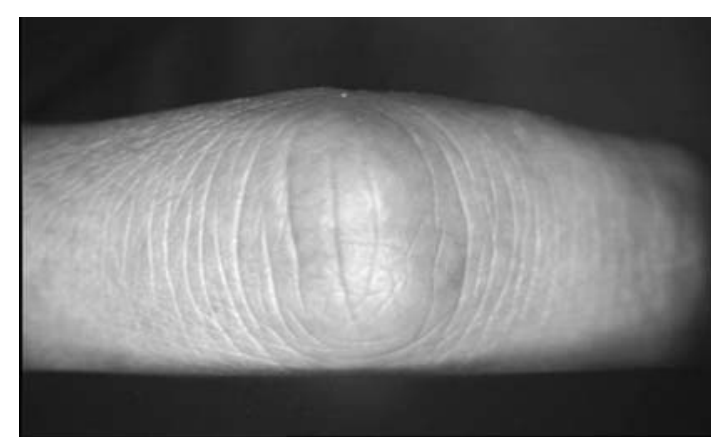

(b)

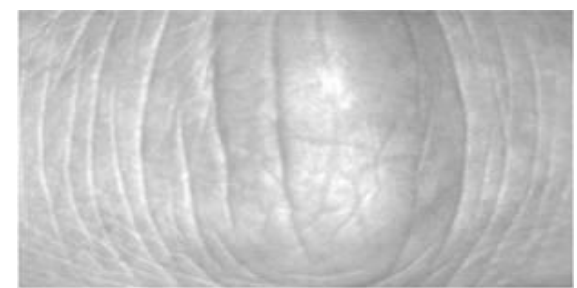

(d)

Fig. 1: (a) Our FKP image acquisition device; (b) a typical FKP image; (c) the determination of ROI; (d) a cropped ROI image from the original FKP image in (c).

In our previous works [18-20], a novel online personal authentication system using finger-knuckle-print (FKP), which refers to the inherent skin pattern of the outer surface around the phalangeal joint of one's finger, has been established. It comprises four major components: FKP image acquisition, ROI (region of interest) extraction, feature extraction and feature matching. The proposed FKP imaging system has a small size and it simplifies the preprocessing steps, such as the finger segmentation and the ROI extraction. Since the finger knuckle will be slightly bent when being 
imaged, the inherent skin patterns can be clearly captured and hence the unique FKP features can be better exploited. Fig. 1a shows the outlook of our FKP image acquisition device and Fig. 1b shows a typical FKP image. Fig. 1c and Fig. 1d illustrate the ROI extraction process presented in [19]. The later feature extraction and matching are based on the extracted ROIs. As in any pattern classification task, the feature extraction and matching plays a key role in our FKP-based personal authentication system. To this end, we have developed a couple of different methods. In [18], we used the Gabor filter based competitive coding scheme, which was originally designed for palmprint recognition [8], to extract the local orientation information as FKP features. In [19], we proposed to combine the orientation information and the magnitude information extracted by Gabor filters together. In [20], the Fourier transform of the whole image was taken as the feature and the band-limited phase-only correlation technique was employed to calculate the similarity between two FKP images.

In this paper, we focus on feature extraction and matching of FKP images. Based on the area of pixels involved in feature extraction, we can label the features as "local" or "global" ones. Intuitively, a local feature is a measure computed within a local patch, encoding the detailed traits within this specific area; by contrast, a global feature is a measure derived from all (or most of) the pixels in the image, reflecting some holistic characteristic of the examined image. According to such definitions, existing FKP recognition schemes can be classified into local-based methods $[15,16,18,19]$ and global-based methods $[17,20]$. However, few papers have yet discussed the local-global information combination for FKP recognition. In the literature of psychophysics and neurophysiology, many studies have shown that both local and global information is crucial for the image perception and recognition of human beings [21] and they play different but complementary roles. A global feature reflects the holistic characteristics of the image and is suitable for coarse representation, while a local feature encodes more detailed information within a specific local region and is appropriate for finer representation. Hence, better recognition accuracy can be expected if local and global information can be appropriately combined.

Such an idea has already been explored in iris recognition, palmprint recognition, face recognition and fingerprint recognition. For iris matching, Sun et al. [22, 23] proposed a "cascade" system in which the first stage is a conventional Daugman-like classifier while the classifier at the 
second stage uses "global" features - areas enclosed by zero-crossing boundaries. In [24], the authors described a two-level palmprint matching scheme. For coarse-level filtering, Hough transform is used to extract global features; for fine-level matching, the local information extracted from the locations and orientations of individual lines is used. Pan et al. also proposed to combine the local and global features for palmprint recognition [25]. In their work, non-negative matrix factorization with sparseness constraint and PCA are used to extract local and global features, respectively. For face recognition, Fang et al. [26] presented a method by combining global PCA features and component-based local features extracted by Haar wavelets. In [21], Su et al. proposed a hierarchical ensemble classifier by combining global Fourier features and local Gabor features. In their method, global features are extracted from the whole face images by keeping the low-frequency Fourier coefficients while local features are exploited using Gabor filters with various scales and orientations. After that, Fisher's linear discriminant (FLD) is applied to the global Fourier features and local Gabor features. In the fingerprint recognition community, the idea of combing local and global information was also exploited [4-6].

In this paper, we propose a novel local-global information combination (LGIC) scheme for FKP recognition. Specifically, we take the local orientation information extracted by the Gabor filters as the local feature because local orientation has been successfully used in palmprint recognition systems $[8,9]$ and FKP recognition systems $[15,18,19]$. By increasing the scale of the Gabor filters, more and more global information will be involved, yet the characterization of image local structures will be weakened rapidly. Particularly, if the scale of the Gabor filter is increased to infinity, the Gabor transform can be reduced to the Fourier transform of the whole image. In this case, no local information can be extracted but we can get the finest resolution for the global frequency analysis of the image. Thus, the Fourier transform coefficients are naturally taken as the global features in this paper. With the global Fourier features, the alignment between intra-class FKP ROIs can also be refined. At the matching stage, two matching distances can be computed by comparing the local features and the global features separately. Finally, the two matching distances are fused according to some fusion rule to get the final matching distance. Extensive experiments and comparisons are conducted on our established FKP database [27] to validate the efficacy of the proposed LGIC 
scheme.

The rest of this paper is organized as follows. Section 2 discusses the extraction of local features and the associated matching metric. Section 3 describes the extraction and the matching of global features. Section 4 presents the LGIC based FKP recognition algorithm. Section 5 reports the experimental results. Finally, conclusions are made in Section 6.

\section{Local Feature Extraction and Matching}

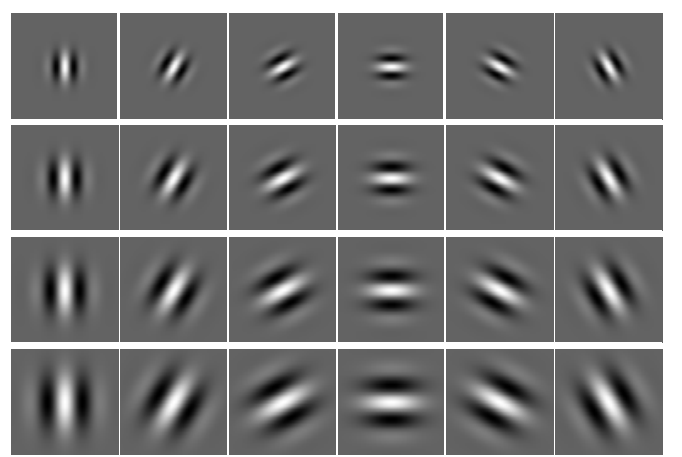

Fig. 2: Real parts of the 24 2D Gabor filters with four scales and six orientations.

Gabor filters [28] have been widely used as an effective tool to fulfill the feature extraction tasks in many biometrics systems, such as face, iris, fingerprint, palmprint, etc. The frequency and orientation representations of Gabor filters are similar to those of the human visual system [29, 30]. In the spatial domain, 2D Gabor filters can be expressed as

$$
G(x, y)=\exp \left(-\frac{1}{2}\left(\frac{x^{\prime 2}}{\sigma_{x}^{2}}+\frac{y^{\prime 2}}{\sigma_{y}^{2}}\right)\right) \cdot \exp \left(i 2 \pi f x^{\prime}\right)
$$

where $x^{\prime}=x \cos \theta+y \sin \theta, y^{\prime}=-x \sin \theta+y \cos \theta$. In Eq. (1), $f$ represents the frequency of the sinusoid factor, $\theta$ represents the orientation of the normal to the parallel stripes of the Gabor function, $\sigma_{x}$ and $\sigma_{y}$ are the standard deviations of the 2D Gaussian envelop.

It can be seen from the definition that a Gabor filter is actually a Gaussian envelop modulated by a sinusoidal plane wave. The Gaussian envelop ensures that the convolution is dominated by the image patch near the center of the filter. Thereby, when an image is convolved with a Gabor filter, the information near the center of the Gaussian envelop is encoded, and by contrast, the information far 
away from the center of the Gaussian envelop will be neglected. Therefore, the Gabor filter is a local operator and can extract the information at a specific scale and a specific orientation within a local region. Gabor filters can have a variety of different forms with different scales and orientations. Fig. 2 shows the real part of the Gabor filters at 4 scales and along 6 orientations.

With the Gabor filters, three basic features, magnitude, phase, and orientation, can be extracted [31]. However, previous studies have shown that the local orientation information is the most robust and distinctive local feature for palmprint and FKP recognition $[8,9,15,18,19]$. Hence, in this paper, we only take the local orientation as the local feature and make use of the Gabor filter based CompCode $[8,18]$ scheme to extract and code it. Such an orientation coding based feature extraction method is suitable for images containing abundant line-like structures and it has the merits of high accuracy, robustness to illumination variation, and fast matching. The working principle of CompCode and its matching metric are briefly reviewed as follows.

Denote by $G_{R}$ the real part of a Gabor filter. With a bank of $G_{R}$ S sharing the same parameters, except the parameter of orientation, the local orientation information of the image $I$ at the position ( $x$, y) can be extracted and coded. Mathematically, this competitive coding process can be expressed as

$$
\operatorname{CompCode}(x, y)=\underset{j}{\arg \min }\left\{I(x, y) * G_{R}\left(x, y, \theta_{j}\right)\right\}
$$

where * stands for the convolution operation, $\theta_{j}=j \pi / J, j=\{0, \ldots, J-1\}$, and $J$ represents the number of orientations. Based on our previous studies $[8,18,19]$, we set $J=6$ in this paper and this is in accordance with the conclusion made by Lee [32] that the simple neural cells are sensitive to specific orientations with approximate bandwidths of $\pi / 6$. Fig. 3c and Fig. 3d show two CompCode maps extracted from the FKP ROI images in Fig. 3a and Fig. 3b, respectively.

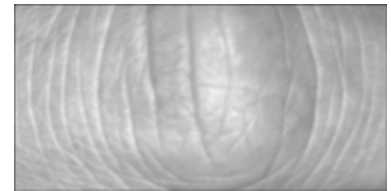

(a)

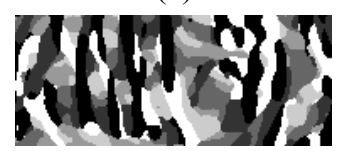

(c)

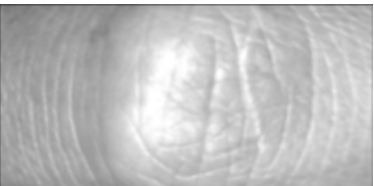

(b)

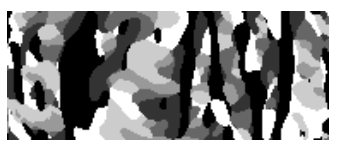

(d)

Fig. 3: (a) and (b) are two FKP ROI images; (c) and (d) are the CompCode maps generated from (a) and (b), respectively. 
In order for real-time recognition, CompCode uses three bits to represent each orientation. When matching two CompCode maps $P$ and $Q$, the angular distance based on the normalized Hamming distance is used [8]:

$$
d_{L}=\frac{\sum_{y=1}^{R o w s} \sum_{x=1}^{C o l s} \sum_{i=0}^{2}\left(P_{i}^{b}(x, y) \otimes Q_{i}^{b}(x, y)\right)}{3 S}
$$

where $P_{i}^{b}\left(Q_{i}^{b}\right)$ is the $i^{\text {th }}$ bit plane of $P(Q), S$ is the area of the CompCode map, and $\otimes$ represents the bitwise "exclusive OR" operation.

\section{Global Feature Extraction and Matching}

\subsection{From local to global}

In section 2, Gabor transforms are utilized to extract the local orientation information. Actually, the Gabor transform can be regarded as a windowed Fourier transform. The corresponding Gabor transform (i.e. filtering) of a function $f$ with respect to a local window function $g$ is [33]

$$
G[f](\mathbf{w}, \mathbf{t})=\int_{-\infty}^{+\infty} \cdots \int_{-\infty}^{+\infty} f(\mathbf{x}) g(\mathbf{x}-\mathbf{t}) e^{-i(\mathbf{w} \cdot \mathbf{x})} d \mathbf{x}
$$

where $\mathbf{t}, \mathbf{w}, \mathbf{x} \in \mathcal{R}^{n}, d \mathbf{x}=d x_{1} d x_{2} \ldots . d x_{n}, \mathbf{x}=\left(x_{1}, x_{2}, \ldots, x_{n}\right)$ and $\mathbf{w} \cdot \mathbf{x}=\sum_{k=1}^{n} w_{k} x_{k}$. The signal $f(\mathbf{t})$ to be analyzed is defined in the $n$-D spatial domain. $\mathbf{t}$ is the coordinate variable in the $n$-D spatial domain and correspondingly, $\mathbf{w}$ is the coordinate variable in the $n$-D frequency domain. The Gabor transform of $f, G[f](\mathbf{w}, \mathbf{t})$ can give the frequency spectrum of $f$ for a specified frequency $\mathbf{w}$ at a specified position

t. For the convenience of discussion, we confine ourselves to the case that $n=1, g$ is a Gaussian-shaped window, and $f(x)$ is of finite length $[0, T]$. Then, the Gabor transform of $f$ is

$$
G[f](\omega, x)=\int_{0}^{T} e^{\frac{-(\tau-x)^{2}}{2 \sigma^{2}}} f(\tau) e^{-i \omega \tau} d \tau, x \in[0, T]
$$

The parameter $\sigma$ controls the size of the local window and the scale of the Gabor transform. Naturally, when $\sigma$ goes to infinity, the whole signal $f(x), x \in[0, T]$, is involved in calculating $G[f](\omega, x)$ and Eq. (5) is reduced to 


$$
G[f](\omega)=\int_{0}^{T} f(\tau) e^{-i \omega \tau} d \tau
$$

It is seen that $G[f]$ does not depend on $x$ anymore, which implies that we lose the local information in the Gabor transform. Obviously, Eq. (6) is the Fourier transform of $f$.

The above discussion on the 1D case can be easily extended to the 2D case. For 2D images, by increasing the scale of the Gabor filters, more and more global information will be involved, yet the characterization of image local structures will be rapidly weakened. Particularly, if the scale of the Gabor filter goes to infinity, the Gabor transform will degrade to the 2D Fourier transform of the whole image. In such case, though the local characterization is totally lost, we can get the finest frequency resolution for the image analysis. Therefore, in our work the Fourier transform is selected as the global feature extractor.

\subsection{Phase-Only Correlation (POC)}

Now that the Fourier transform coefficients are used as the global feature, the next problem is how to measure the similarity of two given Fourier transforms. Phase-Only Correlation (POC) is a classical method to this end [34]. In the literature, POC based methods have been widely used in image registration tasks [35]. Recently, POC has also been adopted as a similarity measure in some biometrics systems [36-38]. Compared with the conventional POC, the Band-Limited Phase-Only Correlation (BLPOC) proposed by Ito et al. [38] is more effective. Hence, in this paper, we use BLPOC to evaluate the displacement parameters between FKP ROIs and to measure the similarity of the Fourier transforms of the aligned ROIs. In this sub-section, POC will be introduced and in the next sub-section BLPOC will be described.

POC is a kind of effective method to evaluate the translation parameters between two images in the Fourier domain. Its underlying principle is the translation property of the Fourier transforms [39]. Let $f$ and $g$ be the two images that differ only by a displacement $\left(x_{0}, y_{0}\right)$, i.e.

$$
g(x, y)=f\left(x-x_{0}, y-y_{0}\right)
$$

Their corresponding Fourier transforms $G(u, v)$ and $F(u, v)$ will be related by 


$$
G(u, v)=e^{-j 2 \pi\left(u x_{0}+v y_{0}\right)} F(u, v)
$$

The cross-phase spectrum $R_{G F}(u, v)$ between $G(u, v)$ and $F(u, v)$ is given by

$$
R_{G F}(u, v)=\frac{G(u, v) F^{*}(u, v)}{\left|G(u, v) F^{*}(u, v)\right|}=e^{-j 2 \pi\left(u x_{0}+v y_{0}\right)}
$$

where $F^{*}$ is the complex conjugate of $F$. By taking inverse Fourier transform of $R_{G F}$ back to the spatial domain, we will have a Dirac impulse centered on $\left(x_{0}, y_{0}\right)$.

In practice, we should consider the finite discrete representations. Consider two $M \times N$ images, $f(m, n)$ and $g(m, n)$, where the index ranges are $m=-M_{0}, \ldots, M_{0}\left(M_{0}>0\right)$ and $n=-N_{0}, \ldots, N_{0}\left(N_{0}>0\right)$, and $M=2 M_{0}+1$ and $N=2 N_{0}+1$. Denote by $F(u, v)$ and $G(u, v)$ the 2D DFTs of the two images and they are given by

$$
\begin{aligned}
& F(u, v)=\sum_{m=-M_{0}}^{M_{0}} \sum_{n=-N_{0}}^{N_{0}} f(m, n) e^{-j 2 \pi\left(\frac{m u}{M}+\frac{n v}{N}\right)}=A_{F}(u, v) e^{j \phi_{F}(u, v)} \\
& G(u, v)=\sum_{m=-M_{0}}^{M_{0}} \sum_{n=-N_{0}}^{N_{0}} g(m, n) e^{-j 2 \pi\left(\frac{m u}{M}+\frac{n v}{N}\right)}=A_{G}(u, v) e^{j \phi_{G}(u, v)}
\end{aligned}
$$

where $u=-M_{0}, \ldots, M_{0}, v=-N_{0}, \ldots, N_{0}, A_{F}(u, v)$ and $A_{G}(u, v)$ are amplitude components, and $\phi_{F}(u, v)$ and $\phi_{G}(u, v)$ are phase components. Then, the cross-phase spectrum $R_{G F}(u, v)$ between $G(u, v)$ and $F(u, v)$ is given by

$$
R_{G F}(u, v)=\frac{G(u, v) F^{*}(u, v)}{\left|G(u, v) F^{*}(u, v)\right|}=e^{j\left\{\phi_{G}(u, v)-\phi_{F}(u, v)\right\}}
$$

The POC function $p_{g f}(m, n)$ is the 2D Inverse DFT (IDFT) of $R_{G F}(u, v)$ :

$$
p_{g f}(m, n)=\frac{1}{M N} \sum_{u=-M_{0}}^{M_{0}} \sum_{v=-N_{0}}^{N_{0}} R_{G F}(u, v) e^{j 2 \pi\left(\frac{m u}{M}+\frac{n v}{N}\right)}
$$

The peak value of $p_{g f}$ can be calculated as $\max \left\{p_{g f}(m, n): \mid m \in\left[-M_{0}, M_{0}\right], n \in\left[-N_{0}, N_{0}\right]\right\}$

If the two images $f$ and $g$ are similar, their POC function $p_{g f}$ will give a distinct sharp peak. If not, the peak value will drop significantly. Thus, the amplitude of the peak value can be used as a similarity measure, and the location of the peak shows the translational displacement between the two images. 


\subsection{Band-Limited Phase-Only Correlation (BLPOC)}

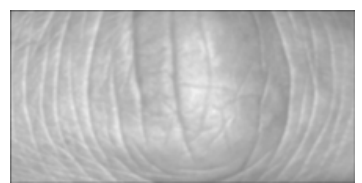

(a)

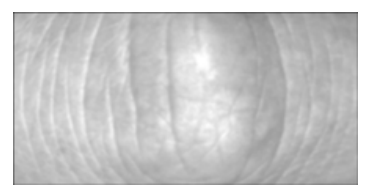

(b)

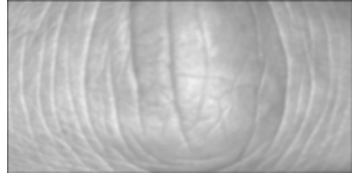

(e)

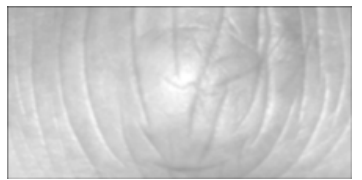

(f)

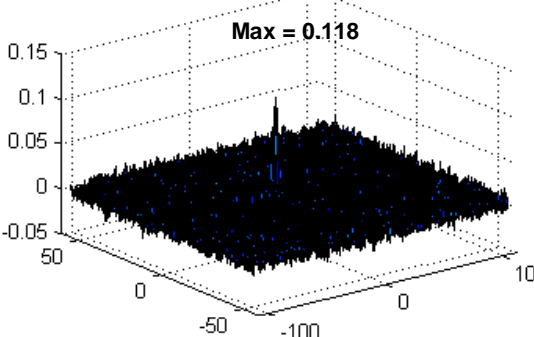

(c)

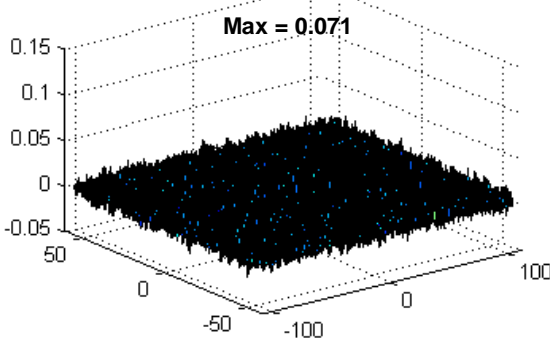

(g)

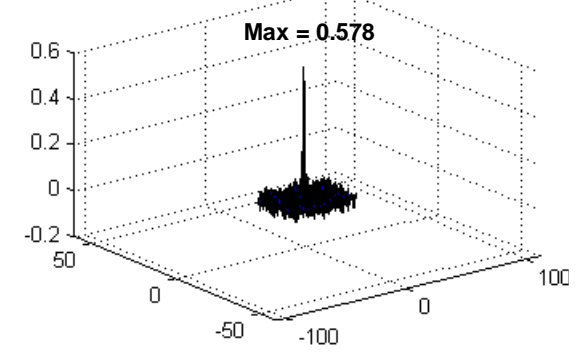

(d)

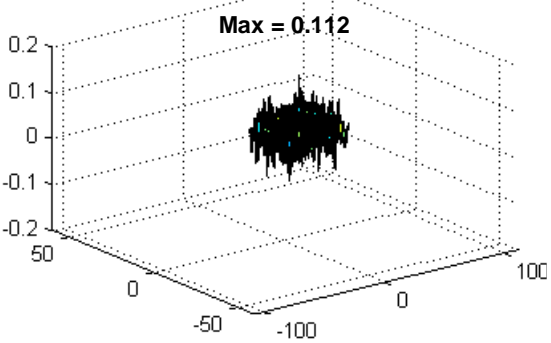

(h)

Fig. 4: Examples of a genuine matching and an imposter matching using POC and BLPOC respectively. (a) and (b) are two FKP ROI images from the same finger, (c) is their POC function and (d) is their BLPOC function. (e) and (f) are two FKP ROI images from different fingers, (g) is their POC function and (h) is their BLPOC function.

In the POC-based image matching method, all the frequency components are involved. However, high frequency components can be prone to noise. To eliminate noisy high frequency components, Ito et al. [38] proposed the Band-Limited POC (BLPOC). BLPOC limits the range of the spectrum of the given FKP image. Suppose that the ranges of the inherent frequency band of FKP texture are given by $u=$ $-U_{0}, \ldots, U_{0}$ and $v=-V_{0}, \ldots, V_{0}$, where $0 \leq U_{0} \leq M_{0}, 0 \leq V_{0} \leq N_{0}$. Thus, the effective size of spectrum is given by $L_{1}=2 U_{0}+1$ and $L_{2}=2 V_{0}+1$. BLPOC function is defined as

$$
p_{g f}^{U_{0} V_{0}}(m, n)=\frac{1}{L_{1} L_{2}} \sum_{u=-U_{0}}^{U_{0}} \sum_{v=-V_{0}}^{V_{0}} R_{G F}(u, v) e^{j 2 \pi\left(\frac{m u}{L_{1}}+\frac{n v}{L_{2}}\right)}
$$

where $m=-U_{0}, \ldots, U_{0}$ and $n=-V_{0}, \ldots, V_{0}$. From the definition of BLPOC, we can see that $U_{0} / M_{0}$ and $V_{0} / N_{0}$ can characterize the inherent frequency distribution of the FKP images.

From the definition of BLPOC, it can be seen that the BLPOC function between two images $f$ and $g$ can be considered as the POC function between their low-pass filtered versions. Thus, the BLPOC 
function can maintain the properties of the POC function. Specifically, if two images are similar, their BLPOC function will have a distinct sharp peak. At the same time, the translational displacement between the two images can be estimated by the location of the peak. Experiments indicate that the BLPOC function provides a much higher discrimination capability than the original POC function in FKP recognition. This can be reflected in the matching examples shown in Fig. 4. Fig. 4a and Fig. 4b are two FKP ROI images from the same finger (captured in different collection sessions), whose POC function and BLPOC function are shown in Fig. 4c and Fig. 4d, respectively; Fig. 4e and Fig. 4f are two FKP ROI images from different fingers, whose POC function and BLPOC function are shown in

Fig. $4 \mathrm{~g}$ and Fig. $4 \mathrm{~h}$, respectively. These examples indicate that in the case of a genuine matching (a matching performed between a pair of FKP images from the same finger), the BLPOC will exhibit a much sharper peak than POC; however, for an imposter matching (a matching performed between a pair of FKP images from different fingers), neither BLPOC nor POC will show a distinct sharp peak. Hence, in this paper, we adopt the BLPOC to align the displacement between FKP ROI images and then to measure the similarity between Fourier transforms of the aligned ROIs.

\section{Local-Global Information Combination (LGIC) for FKP Recognition}

In this section, we present our local-global information combination (LGIC) based FKP recognition algorithm. The entire process of our LGIC-based FKP matching is illustrated in Fig. 5. Given two FKP ROI images $f$ and $g$, the following four steps will be taken to compute their similarity.

\section{Step 1: Translation alignment by global features with BLPOC}

Although the FKP image acquisition device and the ROI extraction algorithm can reduce the geometric transformations between intra-class ROIs much, it is still inevitable that there is some displacement between intra-class ROIs. This will weaken the genuine matching scores. In our previous coding-based works $[18,19]$, this problem was addressed by translating one set of features in horizontal and vertical directions several times and the minimum of the resulting matching distances was considered to be the final matching distance. In this paper, we solve this problem in a different 
way by evaluating the translation parameters between the two ROIs using the BLPOC function. Then we crop the common regions, based on which the feature matching is performed.

The translation parameters $\left(t_{1}, t_{2}\right)$ between $f$ and $g$ can be estimated from the peak location of the global BLPOC of them. Then, we can align $f$ and $g$ based on $\left(t_{1}, t_{2}\right)$ and extract the common regions $f_{C}$ and $g_{C}$. It should be noted that in our system, we will check the ratio between the common region area and the area of the original ROI. If area $\left(f_{C}\right) / \operatorname{area}(f)<t$ (or area $\left.\left(g_{C}\right) / \operatorname{area}(g)<t\right)$, where $t$ is a threshold, $f_{C}$ and $g_{C}$ will be simply set as $f$ and $g$. Generally, this will happen when the two FKP images are from different fingers, i.e. inter-classes.

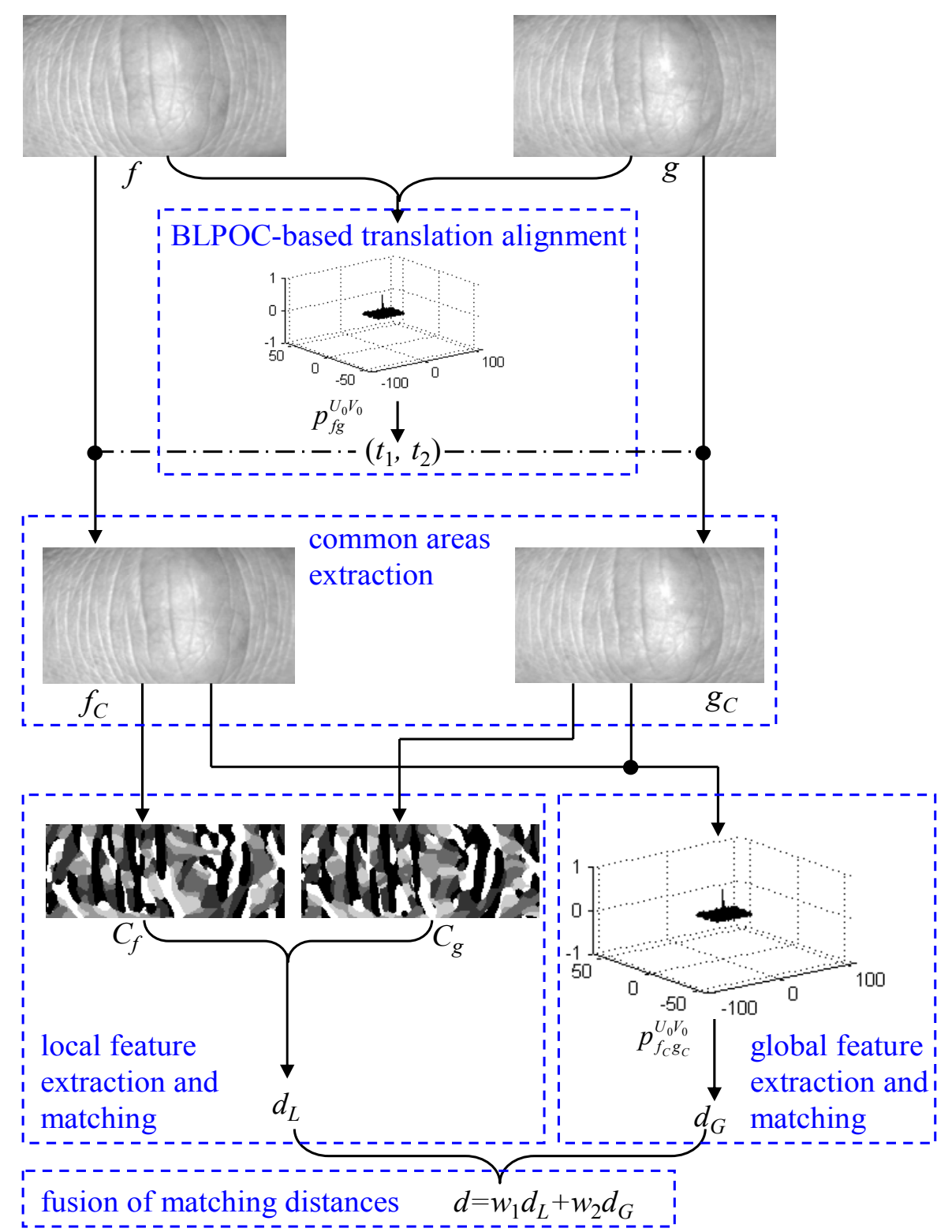

Fig. 5: Illustration for the matching distance computation between a pair of FKP ROI images with LGIC. 


\section{Step 2: Local feature extraction and matching}

After alignment and common area cropping, two CompCode maps $C_{f}$ and $C_{g}$ are constructed from $f_{C}$ and $g_{C}$. Then, by matching $C_{f}$ and $C_{g}$, we could get the matching distance $d_{L}$. For technical details at this step, please refer to Section 2.

\section{Step 3: Global feature extraction and matching}

We use the peak value of the BLPOC function $p_{f g_{c}}^{U_{0} V_{c}}$ between $f_{C}$ and $g_{C}$ to measure the similarity of their Fourier transforms. Denote by pocS the peak value of $p_{f c c c}^{U_{0} V_{0}}$, then the matching distance is defined as: $d_{G}=1-p o c S$.

\section{Step 4: Fusion of matching distances}

Until now, two matching distances $d_{L}$ and $d_{G}$ have been obtained. These two distances can be fused together to get the final matching distance. There are a couple of rules for the fusion of matching distances, such as the Simple-Sum (SS) rule, the MIn-Score (MIS) rule, the MAx-Score (MAS) rule, and the Matcher-Weighting (MW) rule [40]. In our case, $d_{L}$ and $d_{G}$ can be considered to be obtained from two different matchers, matcher 1 (local feature based matcher) and matcher 2 (global feature based matcher), and we adopt the MW rule. With the MW fusion rule, weights are assigned according to the Equal Error Rate (EER) obtained on a training dataset by different matchers. Denote by $e_{k}$ the EER of the matcher $k, k=1,2$. Then, the weight $w_{k}$ associated with matcher $k$ can be calculated as

$$
w_{k}=\left(1 / \sum_{k=1}^{2} \frac{1}{e_{k}}\right) / e_{k}
$$

where $0 \leq w_{k} \leq 1$ and $\sum_{k=1}^{2} w_{k}=1$. It is obvious that the weights are inversely proportional to the corresponding EERs. Then, the final matching distance is calculated as

$$
d=w_{1} d_{L}+w_{2} d_{G}
$$




\section{Experimental Results}

\subsection{FKP database and test protocol}

In our previous work [18-20], an FKP database was established using the developed FKP image acquisition device. This database is intended to be a benchmark to evaluate the performance of various FKP recognition methods, and it is available at [27]. In this database, FKP images were collected from 165 volunteers, including 125 males and 40 females. Among them, 143 subjects were 20 30 years old and the others were $30 \sim 50$ years old. We collected samples in two separate sessions. In each session, the subject was asked to provide 6 images for each of the left index finger, the left middle finger, the right index finger and the right middle finger. Therefore, 48 images from 4 fingers were collected from each subject. In total, the database contains 7,920 images from 660 different fingers. The average time interval between the first and the second sessions was about 25 days. The maximum and minimum time intervals were 96 days and 14 days respectively. In all of the following experiments, we took images collected at the first session as the gallery set and images collected at the second session as the probe set. To obtain statistical results, each image in the probe set was matched with all the images in the gallery set. If the two images were from the same finger, the matching between them was counted as a genuine matching; otherwise it was counted as an imposter matching.

The EER, which is the point where the False Accept Rate (FAR) is equal to the False Reject Rate (FRR), is used to evaluate the verification accuracy. The decidability index $d^{\prime}$ [41] is used to measure how well the genuine and the imposter distributions are separated. $d^{\prime}$ is defined as

$$
d^{\prime}=\frac{\left|\mu_{1}-\mu_{2}\right|}{\sqrt{\left(\sigma_{1}^{2}+\sigma_{2}^{2}\right) / 2}}
$$

where $\mu_{1}\left(\mu_{2}\right)$ is the mean of the genuine (imposter) matching distances and $\sigma_{1}\left(\sigma_{2}\right)$ is the standard deviation of the genuine (imposter) matching distances.

\subsection{Determination of parameters}

In real implementation, parameters need to be determined for LGIC. To this end, we tuned the 
parameters based on a sub-dataset, which contained the first 300 FKP images. Parameters for the local feature and the global feature were tuned separately. Three parameters $\sigma_{x}, \sigma_{y}$, and $f$ need to be tuned for the local feature while two parameters $U_{0} / M_{0}$ and $V_{0} / N_{0}$ need to be tuned for the global feature. The tuning criterion was that parameter values that could lead to a lower EER would be chosen. As a result, the parameters used in this paper were set as: $\sigma_{x}=5.0, \sigma_{y}=9.0, f=0.0435, U_{0} / M_{0}=0.25$ and $V_{0} / N_{0}=0.2$. Moreover, two fusion weights $w_{1}$ and $w_{2}$ can be calculated using Eq. (15).

\subsection{FKP verification results}

Verification aims to answer the question of "whether the person is the one he/she claims to be". In this experiment, all the classes of FKPs were involved. Therefore, there were $660(165 \times 4)$ classes and $3,960(660 \times 6)$ images in the gallery set and the probe set each. Each image in the probe set was matched against all the images in the gallery set. Thus, the numbers of genuine matchings and imposter matchings were 23,760 and $15,657,840$, respectively. In order to show its superiority, the proposed LGIC was compared with the other three state-of-the-art FKP verification methods, CompCode [18], BLPOC [20], and ImCompCode\&MagCode [19]. Some optimizations have been made on ROI extraction and matching, so the experimental results for CompCode, ImCompCode\&MagCode, and BLPOC are better than the previous publications. The results in terms of the EER and $d^{\prime}$ are summarized in Table 1. In addition, the FRRs for each algorithm obtained with a fixed FAR $=1.1 \times 10^{-3}$ are also presented in Table 1 for comparison. Furthermore, by adjusting the matching threshold, a DET (Detection Error Tradeoff) curve [42], which is a plot of False Reject Rate (FRR) against False Accept Rate (FAR) for all possible thresholds, can be created. The DET curve can reflect the overall verification accuracy of a biometric system. Fig. 6a shows the DET curves generated by the four different FKP verification schemes. Distance distributions of genuine matchings and imposter matchings obtained by the proposed LGIC scheme are plotted in Fig. 6b. Table 2 lists five typical operating states obtained by using the LGIC scheme. 


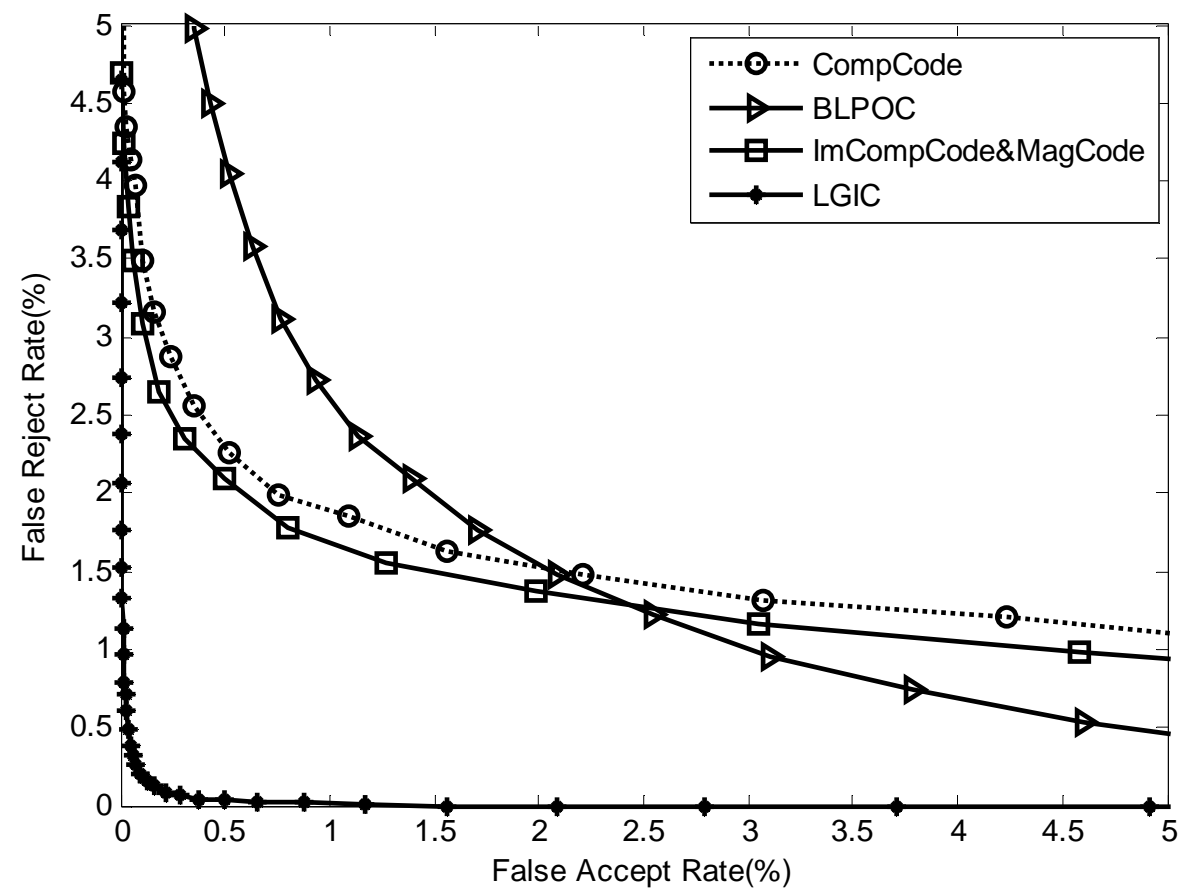

(a)

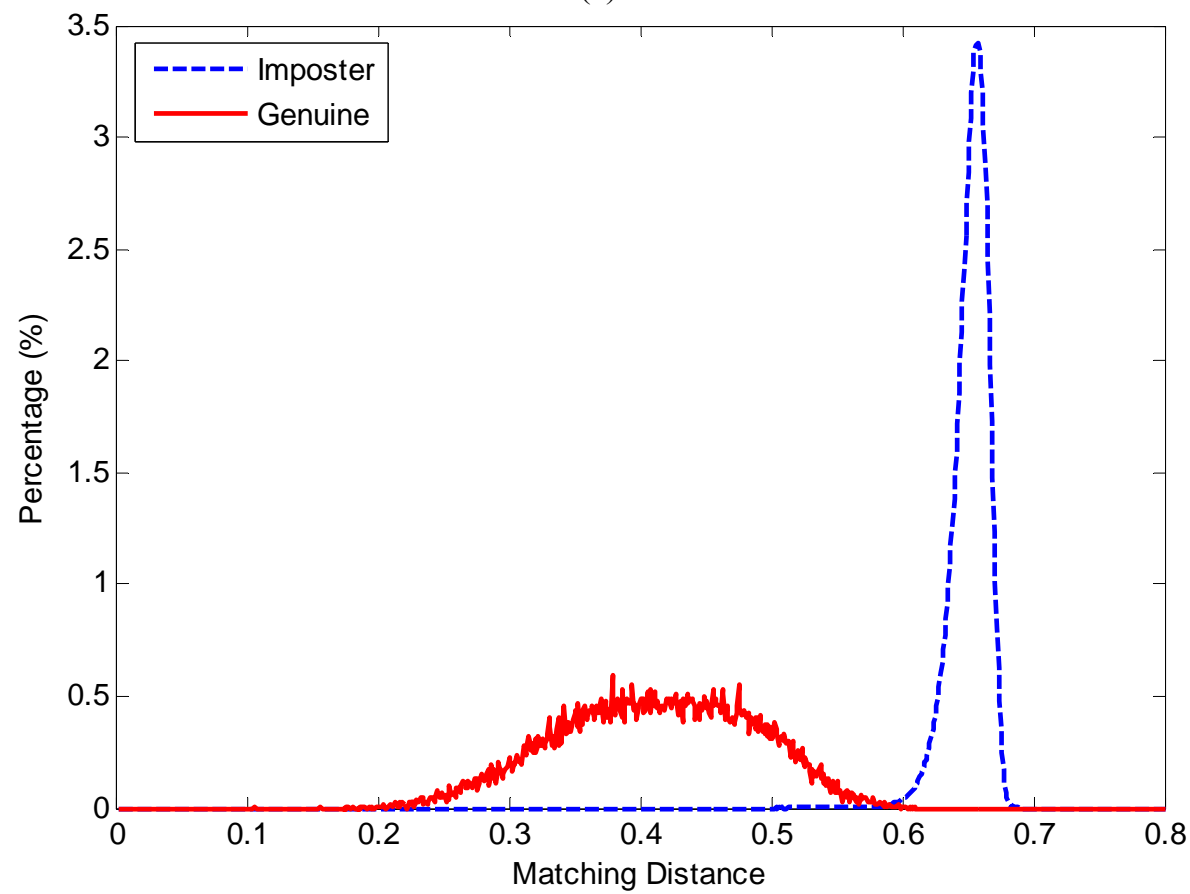

(b)

Fig. 6: (a) DET curves obtained by the four FKP recognition methods; (b) distance distributions of genuine matchings and imposter matchings with the proposed scheme LGIC.

Table 1. Performance comparison of different FKP verification schemes

\begin{tabular}{cccc}
\hline & EER (\%) & $d^{\prime}$ & FRR (\%) $\left(\right.$ when FAR $\left.=1.1 \times 10^{-3}\right)$ \\
\hline CompCode [18] & 1.658 & 4.2989 & 3.4848 \\
BLPOC [20] & 1.676 & 2.4745 & 8.5939 \\
ImCompCode\&MagCode [19] & 1.475 & 4.3224 & 3.0818 \\
LGIC & $\mathbf{0 . 4 0 2}$ & $\mathbf{4 . 5 3 5 6}$ & $\mathbf{0 . 9 6 8 0}$ \\
\hline
\end{tabular}


Table 2. Typical operating states using LGIC

\begin{tabular}{cc}
\hline FAR $(\%)$ & FRR $(\%)$ \\
\hline 0.0515 & 1.5236 \\
0.1068 & 0.9680 \\
0.2148 & 0.6439 \\
0.3396 & 0.4461 \\
0.5982 & 0.2819 \\
\hline
\end{tabular}

From the results listed in Table 1 and the DET curves shown in Fig. 6a, we can see that the proposed LGIC scheme performs significantly better in terms of the verification accuracy than the other state-of-the-art FKP verification methods evaluated, including CompCode, BLPOC and ImCompCode\&MagCode. As stated, the local orientation information or the local magnitude information is used in CompCode and ImCompCode\&MagCode, so they can be classified as local-based methods. By contrast, in BLPOC, the Fourier transform of the whole image is taken as the feature so it is actually a global-based method. Therefore, the experimental results also corroborate the claim that methods fusing local and global information together can outperform the methods depending on only a specific kind of features, local or global.

\subsection{Speed}

Table 3. Computation time for key processes

\begin{tabular}{cc}
\hline Operations & Time $(\mathrm{msec})$ \\
\hline ROI extraction & 198 \\
Translation alignment & 1.4 \\
Competitive coding & 60 \\
Calculation of $d_{L}$ & 0.3 \\
Calculation of $d_{G}$ & 2.1 \\
\hline
\end{tabular}

The FKP recognition software is implemented using Visual C\#.Net 2005 on a Dell Inspiron 530s PC embedded Intel E6550 processor and 2GB of RAM. Computation time for the key processes is listed in Table 3. The execution time for data preprocessing and ROI extraction is $198 \mathrm{~ms}$. The time for BLPOC-based translation alignment is about $1.4 \mathrm{~ms}$. The time for competitive coding is $60 \mathrm{~ms}$. The time for calculating $d_{L}$ and $d_{\mathrm{G}}$ is $0.3 \mathrm{~ms}$ and $2.1 \mathrm{~ms}$, respectively. Thus, the total execution time for one verification operation is less than $0.5 \mathrm{~s}$ in our prototype system, which is fast enough for real-time 
applications. We believe that with the optimization of the implementation, the system's efficiency could be much further improved.

\section{Conclusions}

In this paper, a novel local-global information combination (LGIC) based FKP recognition method was proposed. It is based on the fact that both local and global features are crucial for the image recognition and perception and they play different and complementary roles in such a process. In LGIC, the local orientation extracted by the Gabor filters based competitive coding scheme was taken as the local feature. From the perspective of time-frequency analysis, when the scale of the Gabor transform goes to infinity, it degenerates to the Fourier transform. Thus, the Fourier transform was naturally taken as the global feature in our work. LGIC exploits both local and global features for FKP verification, where the global features were also used to refine the alignment of FKP images in matching. Extensive experimental results conducted on our FKP database indicate that the proposed scheme could achieve much better performance in terms of EER and the decidability index than the other state-of-the-art competitors. Specifically, the EER of LGIC is $0.402 \%$ and it can operate at a low FRR of $1.5236 \%$ with a low FAR of $0.0515 \%$ on our FKP database.

\section{References}

[1] A.K. Jain, P.J. Flynn, A. Ross, Handbook of Biometrics, Springer, 2007.

[2] D. Maltoni, D. Maio, A.K. Jain, S. Prabhakar, Handbook of Fingerprint Recognition, Springer, 2003.

[3] N. Ratha, R. Bolle, Automatic Fingerprint Recognition Systems, Springer, 2004.

[4] J. Gu, J. Zhou, C. Yang, Fingerprint recognition by combining global structure and local cues, IEEE Transactions. Image Processing 15 (7) (2006) 1952-1964.

[5] S.B. Nikam, P. Goel, R. Tapadar, S. Agarwal, Combining Gabor local texture pattern and wavelet global features for fingerprint matching, in: Proceedings of the ICCIMA'07, 2007, pp. 409-416.

[6] J.F. Aguilar, Y. Chen, J. O. Garcia, A.K. Jain, Incorporating image quality in multi-algorithm fingerprint verification, in: Proceedings of the ICB'06, 2006, pp. 213-220.

[7] D. Zhang, W. K. Kong, J. You, M. Wong, Online palmprint identification, IEEE Transactions. Pattern 
Analysis and Machine Intelligence 25 (9) (2003) 1041-1050.

[8] W. K. Kong, D. Zhang, Competitive coding scheme for palmprint verification, in: Proceedings of the ICPR'04, 2004, pp. 520-523.

[9] W. Jia, D. Huang, D. Zhang, Palmprint verification based on robust line orientation code, Pattern Recognition 41 (5) (2008) 1504-1513.

[10] A.K. Jain, A. Ross, S. Pankanti, A prototype hand geometry-based verification system, in: Proceedings of the AVBPA'99, 1999, pp. 166-171.

[11] R. Sanchez-Reillo, C. Sanchez-Avila, A. Gonzalez-Marcos, Biometric identification through hand geometry measurements, IEEE Transactions. Pattern Analysis and Machine Intelligence 22 (10) (2000) $1168-1171$.

[12] J. Wang, W. Yau, A. Suwandy, E. Sung, Personal recognition by fusing palmprint and palm vein images based on “Laplacianpalm” representation, Pattern Recognition 41 (5) (2008) 1531-1544.

[13] Q. Li, Z. Qiu, D. Sun, J. Wu, Personal identification using knuckleprint, in: Proceedings of SinoBiometrics'04, 2004, pp. 680-689.

[14] L. Nanni, A. Lumini, A multi-matcher system based on knuckle-based features, Neural Computing \& Applications 18 (1) (2009) 87-91.

[15] A. Kumar, Y. Zhou, Personal identification using finger knuckle orientation features, Electronic Letters 45 (20) (2009) 1023-1025.

[16] D.L. Woodard, P.J. Flynn, Finger surface as a biometric identifier, Computer Vision and Image Understanding 100 (3) (2005) 357-384.

[17] A. Kumar, C. Ravikanth, Personal authentication using finger knuckle surface, IEEE Transactions. Information Forensics and Security 4 (1) (2009) 98-109.

[18] L. Zhang, L. Zhang, D. Zhang, Finger-knuckle-print: a new biometric identifier, in: Proceedings of the ICIP’09, 2009.

[19] L. Zhang, L. Zhang, D. Zhang, H. Zhu, Online finger-knuckle-print verification for personal authentication, Pattern Recognition 43 (7) (2010) 2560-2571.

[20] L. Zhang, L. Zhang, D. Zhang, Finger-knuckle-print verification based on band-limited phase-only correlation, in: Proceedings of the CAIP'09, 2009, pp. 141-148.

[21] Y. Su, S. Shan, X. Chen, W. Gao, Hierarchical ensemble of global and local classifiers for face recognition, IEEE Transactions. Image Processing 18 (8) (2009) 1885-1896. 
[22] Z. Sun, Y. Wang, T. Tan, J. Cui, Cascading statistical and structural classifiers for iris recognition, in: Proceedings of the ICPR'04, 2004, pp. 1261-1262.

[23] Z. Sun, Y. Wang, T. Tan, J. Cui, Improving iris recognition accuracy via cascaded classifiers, IEEE Transactions. Systems, Man, and Cybernetics-Part C 35 (3) (2005) 435-441.

[24] F. Li, K.H. Leung, X. Yu, A two-level matching scheme for speedy and accurate palmprint identification, in: Proceedings of the MMM'07, 2007, pp. 323-332.

[25] X. Pan, Q. Ruan, Y. Wang, Palmprint recognition using fusion of local and global features, in: Proceedings of the ISPACS'07, 2007, pp. 642-645.

[26] Y. Fang, T. Tan, Y. Wang, Fusion of global and local features for face verification, in: Proceedings of the ICPR'02, 2002, pp. 382-385.

[27] PolyU Finger-Knuckle-Print Database. http://www.comp.polyu.edu.hk/ biometrics/FKP.htm.

[28] D. Gabor, Theory of communication, Journal of the Institute of Electrical Engineers 93 (1946) 429-457.

[29] N. Pinto, J.J. DiCarlo, D.D. Cox, How far can you get with a modern face recognition test set using only simple features?, in: Proceedings of the CVPR'09, 2009, pp. 2591-2598.

[30] N. Pinto, D.D. Cox, B. Corda, D. Doukhan, Why is real-world object recognition hard?: Establishing honest benchmarks and baselines for object recognition, in: Proceedings of the COSYNE'08, 2008.

[31] A. Kong, An evaluation of Gabor orientation as a feature for face recognition, in: Proceedings of the ICPR’08, 2008.

[32] T.S. Lee, Image representation using 2D Gabor wavelet, IEEE Transactions. Pattern Analysis and Machine Intelligence 18 (10) (1996) 957-971.

[33] A.I. Zayed, Handbook of Function and Generalized Function Transformations, CRC Press, 1996.

[34] C.D. Kuglin, D.C. Hines, The phase correlation image alignment method, in: Proceedings of the Int. Conf. Cybernetics and Society’75, 1975, pp. 163-165.

[35] B. Reddy, B. Chatterji, An FFT-based technique for translation, rotation, and scale-invariant image registration, IEEE Transactions. Image Processing 5 (8) (1996) 1266-1271.

[36] K. Miyazawa, K. Ito, T. Aoki, K. Kobayashi, H. Nakajima, An effective approach for iris recognition using phase-based image matching, IEEE Transactions. Pattern Analysis and Machine Intelligence 20 (10) (2008) $1741-1756$.

[37] K. Ito, T. Aoki, H. Nakajima, K. Kobayashi, T. Higuchi, A palmprint recognition algorithm using phase-only correlation, IEICE Transactions. Fundamentals E91-A (4) (2008) 1023-1030. 
[38] K. Ito, H. Nakajima, K. Kobayashi, T. Aoki, T. Higuchi, A fingerprint matching algorithm using phase-only correlation, IEICE Transactions. Fundamentals E87-A (3) (2004) 682-691.

[39] R. Bracewell, The Fourier Transform and Its Applications, McGraw-Hill, 1965.

[40] R. Snelick, U. Uludag, A. Mink, M. Indovina, A.K. Jain, Large-scale evaluation of multimodal biometric authentication using state-of-the-art systems, IEEE Transactions. Pattern Analysis and Machine Intelligence 27 (3) (2005) 450-455.

[41] J. Daugman, The importance of being random: statistical principles of iris recognition, Pattern Recognition 36 (2) (2003) 279-291.

[42] A. Martin, G. Doddington, T. Kamm, M. Ordowski, M. Przybocki, The DET curve in assessment of detection task performance, in: Proceedings of the Eurospeech'97, 1997, pp. 1895-1898. 